Commentary/Mather et al.: Norepinephrine ignites local hotspots of neuronal excitation

BEHAVIORAL AND BRAIN SCIENCES (2016), Volume 39, Pages 1-75

doi:10.1017/S0140525X15000667, e200

\title{
Why we forget our dreams: Acetylcholine and norepinephrine in wakefulness and REM sleep
}

\author{
doi:10.1017/S0140525X15001739, e202, Pages 20-21
}

\section{Andrea Becchetti and Alida Amadeo ${ }^{\mathrm{b}}$}

${ }^{a}$ Department of Biotechnology and Biosciences and Center for Neuroscience of Milan, University of Milano-Bicocca, 20128 Milan, Italy; bDepartment of Biosciences, University of Milano, 20133 Milan, Italy.

andrea.becchetti@unimib.it

http://www.btbs.unimib.it

alida.amadeo@unimi.it

http://www.dbs.unimi.it

Abstract: The ascending fibers releasing norepinephrine and acetylcholine are highly active during wakefulness. In contrast, during rapid-eyemovement sleep, the neocortical tone is sustained mainly by acetylcholine. By comparing the different physiological features of the norepinephrine and acetylcholine systems in the light of the GANE (glutamate amplifies noradrenergic effects) model, we suggest how to interpret some functional differences between waking and rapid-eye-movement sleep.

Regulation of neocortical circuits by ascending regulatory systems involves all of the classic neurotransmitters. Most of the nuclei located in the brainstem, hypothalamus, and basal forebrain not only are reciprocally connected, but also send direct projections to the neocortex (Jones 2011; Saper et al. 2010; Steriade \& McCarley 2005). The same applies to release by the hypothalamic nuclei of neuropeptides such as orexin/hypocretin in wakefulness and melanin-concentrating hormone (MCH) in rapid-eye-movement (REM) sleep (Aracri et al. 2015; Jones \& Hassani 2008;

Monti et al. 2013; and references therein). As a first approximation, these bewildering intricacies can be simplified by focusing on the balance in activity between noradrenergic and cholinergic nuclei, which are crucial regulators of arousal and cognition (e.g., Constantinople \& Bruno 2011; Schmidt et al. 2013). Both project varicose fibers that widely innervate the neocortex, and their global effects are excitatory. During wakefulness, high levels of norepinephrine (NE) and acetylcholine ( $\mathrm{ACh}$ ) cooperate in regulating arousal and cognitive processes. However, although cholinergic transmission is certainly implicated in synaptic plasticity (e.g., Berg 2011), the physiological action of NE is thought to be more persistent and more closely related to memory retention and consolidation (e.g., Constantinople \& Bruno 2011; McGaugh 2013; Schmidt et al. 2013). The activity of noradrenergic and cholinergic neurons decreases during non-REM (NREM) sleep,

whereas in REM sleep, ACh release increases again, whereas NE activity remains low (Datta 2010; Lee et al. 2005; Saper et al. 2010; Takahashi et al. 2010) (Fig. 1). The fact that neocortex activation in REM sleep is sustained mainly by ACh is a further indication that the cholinergic tone is more directly related to consciousness and executive functions. In fact, the role of REM sleep in memory consolidation remains controversial (Ackermann \& Rasch 2014; Rasch \& Born 2013).

Does the GANE model help suggest possible explanations of the different functional consequences of activating these regulatory systems during brain states? A first central assumption is that, under strong neuronal activation, spillover glutamate stimulates nearby $\mathrm{NE}$ varicosities in an $\mathrm{N}$-methyl-D-aspartate (NMDA) receptor-mediated manner. By activating low-affinity $\beta$-adrenoreceptors, high NE release would stimulate neuronal excitability, as well as glutamatergic terminals, thus constituting activity "hotspots" that effectively amplify inputs with high 


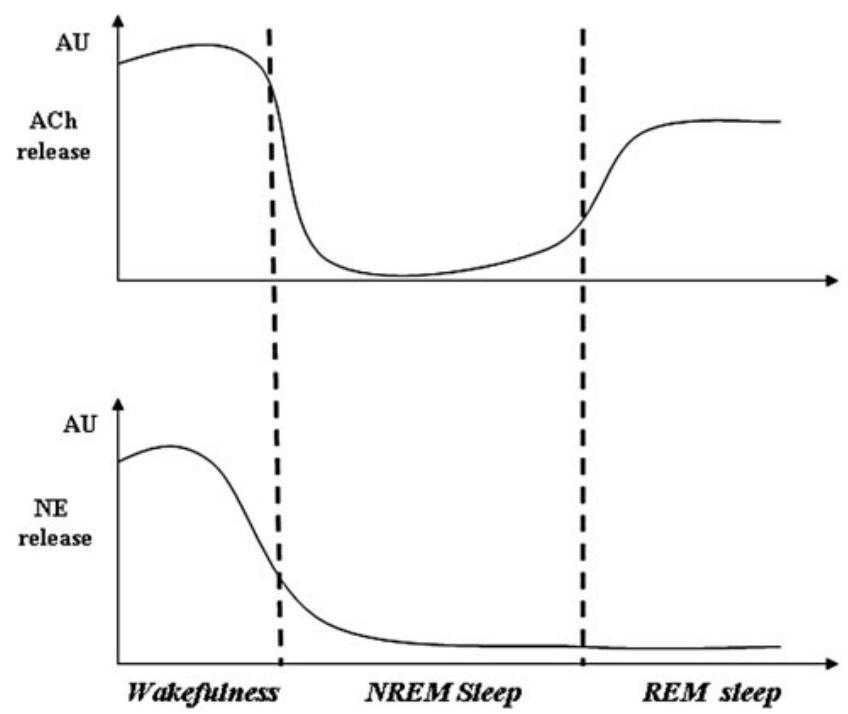

Figure 1 (Becchetti \& Amadeo). Cholinergic and noradrenergic activity through the sleep-wake cycle. The scheme provides a qualitative comparison of the activity of the ascending cholinergic and noradrenergic projections, with no pretension of quantitative precision. $\mathrm{AU}=$ arbitrary units.

priority under phasic arousal. Are such hotspots possible in the cholinergic system? Not much is known about the glutamatergic regulation of ACh release, but evidence does exist of ionotropic glutamate receptors regulating cholinergic terminals in the neocortex (Ghersi et al. 2003; Parikh et al. 2008). Hence, it is conceivable that spillover glutamate also stimulates cholinergic

fibers. Because it is well known that ACh increases glutamate release (Marchi \& Grilli 2010), a positive feedback loop could generate local ACh hotspots, analogous to those hypothesized by Mather and colleagues.

A second tenet of the GANE model is that the low-threshold $\alpha 2$-adrenoreceptors, by responding to low NE concentrations, would inhibit glutamate release in pathways implicated in low priority signaling, under aroused conditions. In this respect, the cholinergic system presents several differences compared with the noradrenergic. In particular: (1) cholinergic fibers form both well-differentiated point-to-point synapses and axon varicosities that sustain diffuse ACh release (Dani \& Bertrand 2007); and (2) ACh activates both metabotropic (muscarinic, mAChRs) and ionotropic (nicotinic, $\mathrm{nAChR}$ ) receptors. In prefrontal regions, M1 mAChRs are widespread and produce excitatory effects related to working memory through different cellular mechanisms (e.g., McCormick \& Prince 1986; Gulledge et al. 2009; Proulx et al. 2014). Their $\mathrm{EC}_{50}$ for $\mathrm{ACh}$ is in the low $\mu \mathrm{M}$ range. On the other hand, nAChRs can be divided into two functional classes (Dani \& Bertrand 2007). Heteromeric nAChRs have high affinity for $\mathrm{ACh}$ (with $\mathrm{EC}_{50}$ in the $\mu \mathrm{M}$ range), relatively low permeability to $\mathrm{Ca} 2+(\mathrm{PCa})$, and slow desensitization in the presence of agonist. Homomeric nAChRs have high $\mathrm{P}_{\mathrm{Ca}}$ (in the order of the one displayed by NMDA receptors), but low affinity for ACh

$\left(\mathrm{EC}_{50} \approx 200 \mu \mathrm{M}\right)$, and quick desensitization kinetics. A striking difference with NE transmission is immediately apparent. The long-term effects on synaptic consolidation are thought to depend on $\mathrm{Ca}_{2}+$ signals. However, within the putative ACh hotspots, the efficacy of high-PCa homomeric receptors would be blunted by quick desensitization. High ACh concentrations would also tend to desensitize heteromeric nAChRs. This would prevent sustained $\mathrm{Ca}_{2}+$ entry through $\mathrm{nAChRs}$ as well as by nAChR-dependent activation of glutamate release, and thus of 
NMDA receptors. Therefore, it seems unlikely that ACh hotspots can produce long-term cellular effects considerably different from those produced by lower ACh concentrations.

In summary, by following up the GANE model reasoning, one is led to conclude that low and high concentrations of NE and ACh produce distinct functional effects on neocortical networks. Low to moderate ACh release sustains global neocortex arousal in both wakefulness and REM sleep. However, in the absence of NE activity (as in REM sleep), cholinergic activity is unable to yield long-term synaptic changes, such as those implicated in memory retention, which would partly explain the well-known difficulty of recalling oneiric activity. Instead, high levels of ACh seem more able to shape the rapid synaptic responses implicated in executive functions, as the quick kinetics of the lowaffinity nicotinic ACh receptors would suggest. We believe that deeper functional studies of the interplay between the ascending regulatory systems, led by heuristic models such as GANE, will greatly lead to progress in understanding the physiological basis of cognition. 